\title{
4 FVG zwischen Grammatik und Phraseologie: Positionen der Forschung
}

\subsection{FVG als Erscheinung der Grammatik}

Hinsichtlich ihrer linguistischen Beschreibung stellen FVG insofern ein erstaunliches Phänomen dar, als sie sowohl in den gängigen phraseologischen Handbüchern des Deutschen als auch in den gegenwartssprachlichen Grammatiken thematisiert werden. Außer den FVG dürfte es jedenfalls kaum einen sprachwissenschaftlichen Gegenstand geben, der sowohl für die Grammatikschreibung als auch für die Phraseologie als relevant betrachtet wird. Diese Ambivalenz deutet bereits darauf hin, dass der Ort der FVG im Sprachsystem alles andere als leicht zu bestimmen ist. Um einer solchen Positionsbestimmung näher zu kommen, sollen zunächst Grammatiken und phraseologische Darstellungen des Deutschen darauf hin befragt werden, aus welchen Gründen bzw. in welchen Zusammenhängen sie FVG thematisieren und wie sie, sofern sie sich explizit dazu äußern, deren Stellung im Sprachsystem bestimmen.

\subsubsection{FVG in Grammatiken des Deutschen}

Zunächst seien die Grammatiken in den Blick genommen. Die Behandlung der FVG in den wichtigsten Grammatiken des Deutschen ist bereits von Heine (2006: 18-44) aufgearbeitet worden, und zwar hauptsächlich im Hinblick auf das bereits in Kapitel 2 behandelte Definitionsproblem. Daher können sich die Ausführungen hier auf die Frage beschränken, welche Eigenschaften von FVG überhaupt Anlass zu der Behandlung in einer Grammatik geben.

Aufschlussreich für mögliche Motive, FVG in Grammatiken zu behandeln, ist in erster Linie der thematische Zusammenhang, in dem FVG in den Grammatiken angesprochen werden. In der IDS-Grammatik (Zifonun/Hoffmann/Strecker 1997) - der umfangreichsten Darstellung der Grammatik des Gegenwarts-deutschen - werden FVG und Verwandtes im Wesentlichen in drei Kontexten thematisiert: im Rahmen der Wortartenlehre, bei der Diskussion des Begriffs ,Prädikat‘ sowie schließlich im Kapitel über Komplemente (vgl. Zifonun/Hoffmann/ Strecker 1997: 53f.; 702-705; 1066-1069). In der Wortartenlehre des genannten Werkes werden FVG neben Modalverben, Hilfsverben und Kopulaverben als

Ә Open Access. (c) 2021 Volker Harm, publiziert von De Gruyter. (c))BY Dieses Werk ist lizenziert unter der Creative Commons Attribution-NonCommercial-NoDerivatives 4.0 Lizenz.

https://doi.org/10.1515/9783110661255-004 
Subklasse der Wortart Verb angesetzt, welche prototypischerweise durch sog. Vollverben wie laufen, schwimmen oder denken repräsentiert ist. Kriterium für den Ansatz der genannten Subklassen ist offenbar die unterschiedliche Weise, in der Modal-, Hilfs- und Funktionsverben an der Bildung von Prädikatsausdrücken beteiligt sind. In ihrem Verständnis von FVG lehnen sich Zifonun et al. dabei eng an die Darstellung bei von Polenz an. So wird die durch von Polenz 1987 geprägte Unterscheidung zwischen Nominalisierungsverb(gefüge) und Funktionsverb(gefüge) übernommen, obwohl diese sich sonst kaum durchgesetzt hat (vgl. van Pottelberge 2007: 440). Im Zusammenhang mit einer ausführlicheren Betrachtung des Prädikatsbegriffs wird dann nochmals auf FVG eingegangen. Sie werden als „einfache Prädikate mit komplexem Ausdruck“ neben idiomatischen Prädikaten behandelt. ${ }^{68}$ Im Kapitel über Komplemente (Zifonun/Hoffmann/Strecker 1997: 1066-1069) wird schließlich dargelegt, dass die NPs und PPs in FVG nur bedingt als Argumente des Prädikats gelten könnten: „[...] ihre nominalen Bestandteile [kann man] bestenfalls als Komplemente ohne Argumentstatus bezeichnen“ (ebd. 1069). Motivation für die Aufnahme von FVG ist somit letztlich das Streben nach einer Klassifikation einzelner grammatischer Phänomene, nämlich der Prädikatsausdrücke sowie der Komplemente. Dass FVG ein Teil der Grammatik als Regelkomponente der Sprache sind, wird hier zumindest nicht explizit behauptet.

Im Zuge der Wortartendarstellung werden FVG auch in der Duden-Grammatik behandelt (Fabricius-Hansen 2005: 424-432). Als definierend für FVG sieht Fabricius-Hansen dabei die Ersetzbarkeit des Gefüges durch ein einfaches Verb an, das dem Nomen des FVG als Ableitungsbasis zugrunde liegt (ebd. 424). Dementsprechend steht in der insgesamt relativ ausführlichen Darstellung das Verhältnis von Gefüge und einfachem Verb sowie die jeweilige Verteilung der Aktanten im Vordergrund. Die Autorin des entsprechenden Kapitels bemerkt hierzu etwa: „Ist das Grundverb intransitiv oder reflexiv, so entspricht das Akkusativobjekt eines FVGs vom Typ (i a) [Verbindungen mit bringen, stellen, setzen, V. H.] semantisch dem Subjekt des Grundverbs“ (Fabricius-Hansen 2005: 430), vgl. (1).

68 Dass FVG bei Zifonun/Hoffmann/Strecker (1997) zu den einfachen Prädikaten gestellt werden, ist wenig überzeugend: Laut von Polenz (1987), an dessen Sichtweise Zifonun et al. sich sonst explizit anlehnen, sind FVG gerade nicht durch eine einfache, sondern durch eine komplexe Prädikatstruktur gekennzeichnet, vgl. von Polenz’ Notierung der kausativen FVG als „BEWIRK $\left(\mathrm{x}_{\mathrm{AG}}, \mathrm{A}(\mathrm{P}(\mathrm{y} . .)).\right)$ “ (1987: 173), welche die beiden Prädikate BEWIRK und P enthält. Wenn kausative lassen- und machen-Prädikate zu Recht zu den komplexen Prädikaten gerechnet werden (ebd. 705f.), erscheint es inkonsequent, kausative FVG unter den einfachen Prädikaten zu behandeln. 
Indem hier die regelmäßige Entsprechung zwischen FVG und etymologisch verwandtem einfachem Verb thematisiert werden, stellt sich diese Darstellung erkennbar in die Tradition von Fillmore (1968: 119f.) und Jackendoff (1974: 418f.), die eine transformationelle Relation zwischen Verb und Nomen-Verb-Verbindung postuliert hatten (z. B. zwischen drink und to have a drink).

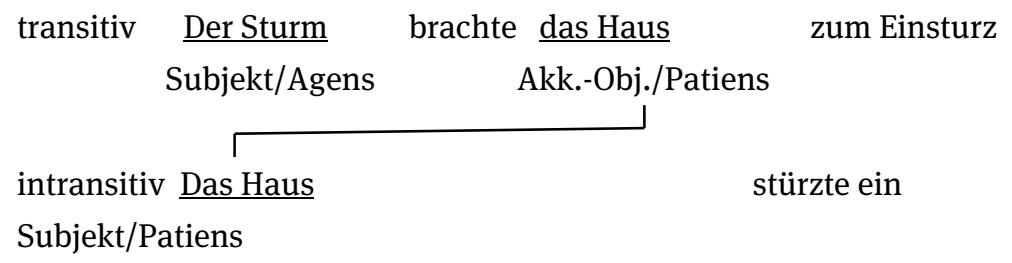

(vgl. Fabricius-Hansen 2005: 430)

Bei Fabricius-Hansen wird mit der Beschreibung solcher Entsprechungen aber offenbar kein reguläres grammatisches Bildungsverfahren postuliert, das auf der Basis eines Verbs eine Nomen-Verb-Verbindung hervorbringt. Vielmehr wird nur ein einzelner grammatischer Sachverhalt - die ansonsten wenig beachtete Relation zwischen FVG und etymologisch verwandtem Verb bei der Aktantenrealisierung - ins Zentrum der Darstellung gerückt. Aus Lernerperspektive mag eine solche Schwerpunktsetzung sinnvoll sein, da die Kenntnis der unterschiedlichen Rollenbesetzungen zum Verständnis der Konstruktionen beitragen kann. Vor dem Hintergrund der in Kapitel 2 diskutierten Definitionsversuche erscheint es freilich eigenwillig, dass die Korrespondenz zwischen FVG und etymologisch verwandtem Verb derart im Mittelpunkt steht, wird das Vorhandensein eines mit dem Nomen im FVG etymologisch verwandten Verbs meist nicht als zentrales Merkmal der Klasse angesehen (s. speziell Kapitel 2.4.1).

In der Grammatik von Eisenberg (2006a: 309-317) werden FVG im Kapitel „Subjekte und Objekte“ behandelt. Eisenberg stellt zunächst mit dankenswerter Klar heit fest: „,Funktionsverbgefüge“ ist mit Sicherheit keine grammatische Kategorie“ (ebd. 309). Der Grund, weshalb er trotzdem relativ ausführlich auf diese Klasse eingeht, liegt primär in der Frage, wie die NPs und PPs syntaktisch zu klassifizieren sind. Als Besonderheit der FVG sieht er folgende Eigenschaft an: „Beim Funktionsver bgefüge bindet sich die Präposition nicht ans Verb, sondern an das Nominal der PrGr. [Präpositionalgruppe, V. H.] [...] Die PrGr insgesamt tritt dann 
in eine syntaktische (und semantische) Beziehung zum Verb und bildet das Funktionsverbgefüge“ (ebd. 301). Damit seien die NPs in FVG sowohl von den Adverbialen als auch von den Präpositionalobjekten abgrenzt. ${ }^{69}$

Bei Helbig/Buscha (2001: 70-83) findet sich die wohl ausführlichste zusammenhängende Darstellung von FVG in einer Grammatik (und zwar innerhalb des Abschnitts „Klassifizierung der Verben nach semantischen Kriterien“, das zum Kapitel „Verb“ gehört). Helbig/Buscha bieten eine Definition des Begriffs (im Anschluss an Helbig 1984) sowie eine nach den einzelnen Verben geordnete umfängliche Bestandsaufnahme der FVG. Sie gehen ferner auf syntaktische Probleme wie Artikelgebrauch, Attribuierbarkeit der NP/PP, Negation und anders mehr ein. Die verhältnismäßig breite Darstellung dieses Gegenstandsbereichs bei Helbig/Buscha ist nicht zuletzt darauf zurückzuführen, dass das Werk speziell für den Unterricht von Deutsch als Fremdsprache konzipiert ist. In diesem Kontext ist eine detaillierte Darstellung dieser sicher schwer zu lernenden Gruppe in der Tat angezeigt. Eine deutliche Aussage dazu, ob FVG selbst Teil der Grammatik sind oder nicht, findet sich hier freilich nicht.

Einen Gegenpol zu den bisher behandelten Grammatiken bildet die ,Textgrammatik der deutschen Sprache‘ von Weinrich (1993). In Weinrichs Darstellung werden FVG zwar ebenfalls im Zusammenhang mit der Wortartenlehre thematisiert; die Behandlung der FVG als grammatisches Phänomen ist hier aber vor allem dadurch motiviert, dass der Autor FVG als ,äußerst produktives Wortbildungsverfahren“ ansieht (Weinrich 1993: 1054). Dementsprechend weicht auch seine Sicht auf die Bildungsweise von FVG von den üblichen Beschreibungen ab: Statt von ,FVG' spricht er von ,Funktionsverben“ als komplexen Wortbildungseinheiten, die aus zwei Teilen bestehen: einem sog. ,Vorverb“ und einem ,Nachverb“ (Weinrich 1993: 45). Das Vorverb entspricht dem geläufigen Begriff ,FV‘, das Nachverb ist die NP bzw. PP. Die Funktion, die Weinrich bei seiner Behandlung der FVG in der Vordergrund rückt, ist daher naheliegender Weise auch eine grammatische, nämlich die Bildung von Formulierungsalternativen für das Passiv (Das Stück kommt zur Aufführung neben dem Ausdruck mit Passivauxiliar Das Stück wird aufgeführt).

Lässt man die hier nur abrissartig vorgestellte Behandlung von FVG in großen Grammatiken des Deutschen Revue passieren, so ist als weitreichende Gemeinsamkeit festzuhalten, dass FVG bzw. vor allem die FV im Rahmen der Wortartenlehre thematisiert sind. FV werden dabei als besonderer Subtyp der Wortart ,Verb“ behandelt, der zwischen ,Vollverb“ und ,Hilfsverb“ angesiedelt wird (so am deutlichsten bei Zifonun/Hoffmann/Strecker 1997). Eine genaue Begründung

69 Eine eingehende Erörterung dieses Abgrenzungsproblems bietet Kapitel 3. 
dieser Zuordnung - etwa anhand des syntaktischen Verhaltens der jeweiligen Verben, wie sie zuletzt Butt/Lahiri (2013) vorgelegt haben - wird nicht geboten (dazu auch Kapitel 8.4.1).

Sieht man von der Frage der Wortartenklassifikation ab, bleibt festzuhalten, dass die einzelnen Grammatiken oft deutlich unterschiedliche Schwerpunkte in ihrer Beschreibung setzen. Die Aktantenverteilung zwischen FVG und einem korrespondierenden einfachen Verb spielt lediglich in der Duden-Grammatik eine herausgehobene Rolle. Bei Eisenberg hingegen steht das Verhältnis der in FVG enthaltenen NPs und PPs in FVG zu den Präpositionalobjekten und Adverbialen im Vordergrund. Bei Helbig/Buscha hingegen wird auch das Problem der Definition von FVG ausführlich behandelt.

Vor allem aber fällt auf, dass die Grammatikalität der FVG selbst unterschiedlich beurteilt wird. Fabricius-Hansen (2005) in der Duden-Grammatik und - sehr dezidiert - Weinrich (1993) sehen in FVG eine regelhafte und produktive Erscheinung, einen Mechanismus, der einfache Verben und Nomen-Verb-Gefüge, welche ein von dem Verb abgeleitetes Nomen enthalten, in eine systematische Beziehung setzt. Sie kommen damit den in der Tradition von Fillmore (1968) und Jackendoff (1974) stehenden Analysen zu den englischen Pendants von FVG am nächsten, welche diese als systematisch gebildete Verbalperiphrase beschreiben (Wierzbicka 1988; Dixon 1991: 336-362; vgl. auch Allerton 2001: 16f.). Auf der anderen Seite steht vor allem die Grammatik von Eisenberg. Dieser erörtert zwar grammatische Phänomene, die mit FVG verbunden sind, und zieht zumindest als Möglichkeit in Betracht, dass es sich bei FV um eine eigene „Wortkategorie neben den Vollverben, Kopulaverben und Modalverben“ handelt (Eisenberg 2006a: 309). FVG selbst werden aber ausdrücklich nicht als grammatische Kategorie angesehen (ebd.). Somit lassen die hier referierten FVG-Kapitel in den Grammatiken des Deutschen die Stellung der FVG im Sprachsystem letztlich offen: Es bleibt unentschieden, ob es sich um ein Phänomen der ,Speicherkomponente‘, also des Lexikons, und vielleicht sogar um Idiome handelt oder ob FVG regelhaft sind und damit zur Grammatik gehören. Über die Position von FVG im Sprachsystem lässt sich daher an dieser Stelle nicht viel mehr sagen, als dass diese in „einem umstrittenen Grenzbereich zwischen Syntax und Phraseologie“ anzusiedeln sind (van Pottelberge 2007: 436; vgl. Fleischer 1997: 131).

\subsubsection{FVG in der Grammatikalisierungsforschung}

Ein methodisches Instrument, das den Anspruch erhebt, die Grammatikalität einer sprachlichen Erscheinung, genauer gesagt, deren Position auf einer zwischen 
den Polen Lexikon und Grammatik gespannten Skala $\mathrm{zu}$ bestimmen, hat die Grammatikalisierungsforschung entwickelt (grundlegend Lehmann 1995; Hopper/Traugott 2003). Die Grammatikalisierung lexikalischer Einheiten ist zwar in erster Linie eine Erscheinung des Sprachwandels; wie jede sprachliche Entwicklung schlägt sie sich auch in synchronen Gegebenheiten wieder. Insofern vermag die Grammatikalisierungsforschung auch Übergänge und Stufen zwischen Lexikon und Grammatik innerhalb eines Sprachzustandes zu beschreiben (vgl. Hopper/Traugott 2003: 2). Daher ist in unserem Zusammenhang auch von dieser Seite eine Klärung der Position von FVG im Sprachsystem zu erwarten.

Im Zusammenhang mit der Beschreibung von Grammatikalisierungserscheinungen in der deutschen Gegenwartssprache hat Lehmann (1991) u. a. FV bzw. FVG behandelt. Maßgeblich für seine Einordnung der FVG sind offenbar vor allem zwei Gesichtspunkte aus dem üblicherweise angewandten Kriterienset für Grammatikalisierung (dazu Lehmann 1995: 126f.; Hopper/Traugott 2003: 100106, 159-165): Die Desematisierung sowie die Paradigmatisierung des Verbs. Die Desemantisierung beschreibt Lehmann im Großen und Ganzen in der bereits aus Kapitel 2 bekannten Weise: Das Verb trägt alle grammatischen Funktionen, während alle lexikalischen Inhalte im Nomen aufgehoben sind (Lehmann 1991: 518). Als grammatische Funktionen wird dabei nicht nur das Inventar der grammatischen Kategorien Person, Numerus, Genus verbi, Tempus sowie Aktionsart angesehen, sondern, nach den Beispielen zu schließen, auch Kausativität. Im Hinblick auf dieses Kriterium wären somit alle FV, die üblicherweise in der Forschung behandelt worden sind, grammatikalisiert.

Das Kriterium der Paradigmatizität lässt freilich unterschiedlich stark grammatikalisierte FVG zu Tage treten: Zum einen gibt es, so Lehmann, Fügungen, die schwach grammatikalisiert seien (Lehmann nennt keine Beispiele), die somit als Idiome im Lexikon gespeichert sind. Zum anderen werden Verbindungen angesetzt, die sich zu größeren Paradigmen zusammenschließen, wie Tab. 2 zeigt, und die daher als stärker grammatikalisiert gelten können.

Tab. 2: FV im Paradigma (nach Lehmann 1991: 519)

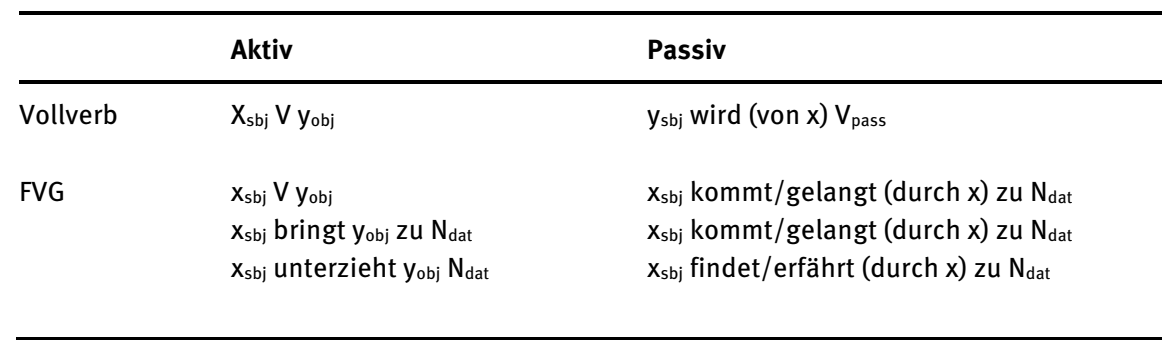


Mit der Betonung der Paradigmatizität zumindest einer größeren Gruppe von FVG stellt sich Lehmann ganz in die Tradition von Polenz' und Helbigs, die in dem Vorhandensein systematischer Oppositionsbeziehungen (z. B. zwischen bringen, kommen und sein) ein zentrales Merkmal von FVG erblickt hatten (s. Kapitel 2.2.4.2). Der Einwand, der oben gegen die FVG angeblich kennzeichnende Oppositionsbildung vorgebracht wurde, gilt indes auch hier: Oppositionen bzw. Paradigmen dieser Art treten auch bei Phraseologismen (aus dem Häuschen bringen/geraten/sein) sowie bei Vollverben auf (ins Krankenhaus kommen/bringen/im Krankenhaus sein; van Pottelberge 2001: 231). Es liegt somit keine spezifische Eigenschaft von FVG vor.

Ein gewichtiges Argument dafür, dass es sich bei FVG - sieht man von idiomatischen Fällen wie eine Entscheidung treffen ab - wenigstens teilweise um eine Erscheinung der Grammatik handelt, ist Lehmann zufolge die Produktivität des Bildungsmusters in Tab. 2: ,The whole strategy [...] is productive more or less like a derivative pattern“ (1991: 518). Hinter Lehmanns Einschränkung - „more or less like a derivative pattern“ - verbirgt sich freilich eine Reihe von Fragen: Während im Gegenüber von aktivem bringen und passivischem kommen tatsächlich ansatzweise ein produktives Muster wie in Tab. 2 greifbar ist, besteht zwischen unterziehen und finden/erfahren eine deutlich schwächer ausgeprägte Korrelation. Kommen-Fügungen haben zwar überwiegend eine Entsprechung mit bringen, einer Konstruktion mit unterziehen stellt sich jedoch nicht automatisch eine mit erfahren/finden an die Seite, vgl. (2) und (3).

(2) a. Dieser Gedanke erfährt ausreichende Beachtung durch Peter.

b. *Peter unterzieht den Gedanken einer ausreichenden Beachtung.

(3) a. Dieser Gedanke findet Berücksichtigung (durch Peter).

b. `Peter unterzieht den Gedanken einer Berücksichtigung.

In diesem Fall bestehen somit sehr deutliche Beschränkungen für das von Lehmann angenommene „derivative pattern“, das erfahren-Fügungen aus Verbindungen mit unterziehen bilden soll. Diese Beschränkungen sind im Einzelnen zu ermitteln, bevor Aussagen über Produktivität möglich sind. 


\subsection{FVG in der Phraseologie}

\subsubsection{Die Kategorie ,Phraseologismus‘}

In gängigen phraseologischen Handbüchern des Deutschen, etwa Burger/Buhofer/Sialm (1982), Fleischer (1997) und Burger (2010), werden FVG zwar behandelt, sie zählen hier jedoch ausnahmslos nicht zum Kernbestand der Phraseologie als Kern gelten allgemein sog. ,vollidiomatische“ Wendungen wie an jmdm. einen Narren fressen oder ins Gras beißen, deren Bedeutung nicht oder nur eingeschränkt aus den Bedeutungen ihrer Teile abgeleitet werden kann und die daher als prinzipiell nicht-kompositional anzusehen sind (zu diesem zentralen Merkmal von Phraseologismen Dobrovol'skij 1995: 28f., 47; Nunberg/Sag/Wasow 1994: 492, 498; Farø 2015: 231). Die randständige Position der FVG innerhalb der Phraseologie wird auch deutlich, wenn man sich noch einmal die Kriterien vergegenwärtigt, die üblicherweise für die Definition von Phraseologismen (bzw. „idioms“ in der angelsächsischen Beschreibungstradition) herangezogen werden. Neben der eben erwähnten Nicht-Kompositionalität sind dies nach Nunberg/Sag/Wasow (1994: 492f.) die folgenden Aspekte:

- Konventionalität: die Auswahl der lexikalischen Elemente ist fest (jmdm. einen Bären aufbinden und nicht z. B. *jmdm. einen Bären umhängen),

- Eingeschränkte morphosyntaktische Flexibilität: Phraseologismen weisen ein reduziertes Paradigma auf und sind typischerweise nicht frei konstruierbar (Sie hat einen Narren an dem Kind gefressen - 'Sie frisst einen Narren an dem Kind; Das ist kalter Kaffee - \#Das ist Kaffee, der kalt ist),

- Bildlichkeit: schwarzes Schaf, jmdm. ein Ohr abkauen, rot sehen,

- Soziale Relevanz: Phraseologismen drücken relevante Konzepte des menschlichen Zusammenlebens aus - ,a recurrent situation of particular social interest" (Nunberg/Sag/Wasow 1994: 493). Dies sind etwa Tabubereiche (ins Gras beißen) oder anderweitig markierte Domänen wie starke Emotionen (auf Wolke Sieben schweben), Geld (die Kröten zusammenhalten), Intelligenz bzw. Dummheit (schlau wie ein Fuchs, dumm wie Brot), normabweichendes Verhalten (jmdm. ein Ohr abkauen) oder Wahnsinn (nicht mehr alle Latten am Zaun haben) usw.,

- Expressivität: Phraseologismen drücken das Gemeinte nicht neutral aus, sondern lassen Wertungen der Sprecher erkennen und erzeugen in der Regel eine besondere Aufmerksamkeit,

- Kolloquiales Stilniveau: Phraseologismen gehören eher der Umgangssprache als der Schriftsprache an. 
Nunberg/Sag/Wasow räumen zwar ein, dass abgesehen von der Konventionalität der Verbindung keine der anderen Eigenschaften obligatorisch für einen Phraseologismus ist; gleichwohl ist mit dieser Liste von Eigenschaften ein prototypischer Bereich abgesteckt. Dass FVG nicht in diesen prototypischen Bereich gehören, ergibt sich schon daraus, dass eigentlich nur die ersten beiden (und gleichzeitig allgemeinsten) Eigenschaften für sie relevant sind: FVG sind insofern konventionalisierte Verbindungen, als das Verb in der Regel feststeht, wie (4) illustriert, und sie zeigen insofern eine eingeschränkte syntaktische Flexibilität, als sie teilweise nicht attribuierbar sind, vgl. (5).

(4) zur Aufführung bringen/\#transportieren/\#mitschleppen/\#holen

(5) *zur gelungenen Aufführung bringen

Das Kriterium der Konventionalität bereitet aber Probleme, da die N-Position eines FVG wesentlich freier besetzt werden kann als dessen Verbposition, vgl. die Vielzahl der Einsatzmöglichkeiten in (6).

(6) zur Anwendung/Anzeige/Aufführung/Ausstrahlung/Durchführung/Verteilung/Versteigerung/Vollendung bringen (s. auch Kapitel 5.2)

Die anderen der genannten Kriterien für Phraseologismen treffen auf FVG jedenfalls, wie angedeutet, nicht oder nur sehr bedingt zu: Die Bildlichkeit von FVG ist gegenüber typischen Phraseologismen wie einen Narren an jmdm. fressen wenig ausgeprägt. FVG stehen ganz überwiegend auch nicht für sozial besonders relevante Situationen, sie dienen nicht dem Ausdruck von Affekten und Bewertungen, und sie sind nicht spezifisch umgangssprachlich, sondern zum Teil sogar eher formell bzw. schriftsprachlich (Farø 2015: 234). Dass FVG in den Randbereich einer Kategorie ,Phraseologismus‘ gehören, liegt somit auf der Hand..$^{70}$ Das heißt aber auch, dass es wegen der starken Einschränkungen bei der Besetzung der Verbposition, wie sie z. B. in (4) vorliegt, unangebracht ist, sie gänzlich von den Phraseologismen zu trennen, wie Palm (1995: 2) vorschlägt. Eine Konventionalisierung ist hier sehr wohl erkennbar.

Da eine Einordung der FVG irgendwo am Rande der Kategorie ,Phraseologismus' aber wenig befriedigend ist, hat sich die Forschung um eine genauere Posi-

70 Auch wenn man andere Kriterienlisten zugrunde legt, wie etwa die von Dobrovol'skij (1995: 27-48), Fleischer (1997: 47-58) oder Sailer (2003: 247-251), kommt man zu einem ähnlichen Ergebnis. Auf die genannten Klassifikationen sei daher nicht näher eingegangen. 
tionsbestimmung bemüht. Hier sind im Wesentlichen zwei Lösungswege beschritten worden: Zum einen werden FVG den Kollokationen zugeschlagen bzw. in deren Nähe gerückt, zum anderen werden sie als sog. „Phraseoschablonen“ behandelt. Diese beiden Optionen werden im Weiteren eingehender geprüft. Im Anschluss daran werden einige wichtige Parameter der Phraseologieforschung Kodierungs- vs. Enkodierungsidiome, Teilbarkeit vs. Nicht-Teilbarkeit - im Hinblick auf ihre Eignung für die genauere Einordnung von FVG durchgespielt.

\subsubsection{FVG als Phraseoschablonen}

Die These, dass FVG unter die Phraseoschablonen fallen, wird in erster Linie von Fleischer (1997: 134-138) vertreten (vgl. auch Fleischer 2001: 116f.). Phraseoschablonen sind nach Fleischer (1997: 131f.) Verbindungen eines invariablen Synsemantikons ${ }^{71}$ (Präposition, Konjunktion) mit einer oder mehreren Leerstellen für im Prinzip beliebiges lexikalisches Material. Phraseoschablonen sind damit ,syntaktische Strukturen [...], deren lexikalische Füllung variabel ist, die aber eine Art syntaktischer Idiomatizität aufweisen“ (1997: 131; vgl. auch den verwandten Begriff der „Modellbildung“ bei Häusermann 1977: 30ff. und Burger 2010: 44f. sowie das Konzept des „formal idiom“ bei Fillmore/Kay/O`Connor 1988: 505f.). Mit dem Ansatz der Kategorie Phraseoschablone wird somit ein „Grenzbereich der Phraseologie zur Syntax“ (Fleischer 1997: 131) in den Blick genommen.

Als typisches Beispiel für eine Phraseoschablone sei die Kombination „N für N“ genannt (vgl. auch Jackendoff 2008 für das Pendant im Englischen):

(7) a. Es geht Schritt für Schritt voran.

b. Ich werde Tag für Tag unruhiger.

c. Glas für Glas wurde er immer redseliger.

d. Mit Beharrlichkeit und seiner scheinbar grenzenlosen Geduld überwand er Krise für Krise (Nürnberger Nachrichten, 29.12.2008, S. 25; Zugriff 06.04.2021)

Die von Fleischer angesprochene „syntaktische Idiomatizität“ dieser Strukturen besteht darin, dass deren Bedeutung nicht unmittelbar aus den regulären Bedeu-

71 Zum Begriff „Synsemantikon“ einführend Harm (2015: 17). 
tungen ihrer Bestandteile herleitbar ist. So kann die Bedeutung der Phraseoschablone N für N, die Fleischer (1997: 132) vage als Intensivierung bzw. Iteration beschreibt, nicht direkt aus den Bedeutungen ihrer Teile rekonstruiert werden.

Prototypische Fälle von Phraseoschablonen sind neben dem eben aufgeführten Muster N für $\mathrm{N}$ etwa auch die intensivierende Wiederholung des Verbs mit eingeschaltetem und (Der Wagen will und will nicht anspringen), die bekräftigende Formel $X$ ist $X$ (Arbeit ist Arbeit, vorbei ist vorbei, gegessen ist gegessen) oder $\mathrm{N}$ hin, N her. Auch expressive Formeln wie z. B. Der und studieren! - die sog. ,incredulity response construction“ (Lambrecht 1990) - rechnet Fleischer (1997: 133) zu den Phraseoschablonen.

Hinsichtlich der Variabilität der in die jeweilige Leerstelle einzutragenden Lexeme bestehen allerdings erhebliche Unterschiede zwischen den einzelnen Schablonen. Im Gegensatz zu den bisher genannten Verbindungen, die hinsichtlich ihrer lexikalischen Füllung offenbar nur wenigen Einschränkungen unterliegen, bestehen bei anderen Mustern deutlich weniger Variationsmöglichkeiten. So stellt nach Fleischer (1997: 133; 2001: 116) auch die sog. „Potenzierungsformel“ Det $+\mathrm{N}+$ von + Det $_{\text {indef }}+\mathrm{N}$ (ein Betonklotz von einem Hotel, dieses Bierfass von einem Kerl) eine Phraseoschablone dar. Die vorliegenden Leerstellen sind, wie (9c) und (9d) belegen, offenbar nicht derart leicht zu füllen wie im Fall von $\mathrm{N}$ für $\mathrm{N}$ in (8).

(8) Tag für Tag, Schritt für Schritt, Teller für Teller, Auto für/um Auto, Klavier für Klavier, Krise für Krise, Programm für Programm

(9) a. ein Betonklotz von einem Hotel

b. ein Mäuseloch von einem Hotel

c. ?ein Reihenhaus von einem Hotel

d. ?ein Einfamilienhaus von einem Hotel

In die Schablone in (9) können offenbar nur Lexeme eintreten, die eine expressive Konnotation aufweisen und ein Extrem - etwas sehr Großes oder sehr Kleines - ausdrücken. Damit ist die Variabilität dieses Musters gegenüber derjenigen in (8) begrenzt. Dies gilt auch für die bei Fleischer (1997: 133) ebenfalls als Phraseoschablone geführte Struktur Es ist zum + V. Die freie Position kann hier nur durch eine begrenzte Zahl von Verben eingenommen werden, nämlich lediglich durch solche, die in irgendeiner Weise eine starke Emotion ausdrücken, wie die Opposition von (10a) und (10b) zeigt. 
(10) a. Es ist zum Heulen, Davonlaufen, Schreien, Verrücktwerden!

b. ^Es ist zum Sprechen, Gehen, Essen!

Zur Klasse der Phraseoschablonen, wie sie maßgeblich von Fleischer postuliert worden ist, bleibt somit festzuhalten, dass hier unterschiedlich starke Beschränkungen hinsichtlich der Besetzbarkeit der jeweiligen Leerstellen gegeben sind. Es handelt sich weniger um eine fest umgrenzte Klasse als vielmehr um ein Kontinuum, das von stark schematischen Strukturen mit relativ freier lexikalischer Füllbarkeit bis hin zu weniger schematischen Strukturen mit eingeschränkter Variabilität reicht.

In FVG sieht Fleischer (1997: 135) einen „Spezialfall der Phraseoschablonen“. Eine Analogie zwischen Phraseoschablonen und FVG besteht Fleischer zufolge zunächst darin, dass auch in FVG das Verb als konstantes lexikalisches Element mit einem variabel einsetzbaren Verbalnomen kombiniert ist, vgl. die Verbindungen mit kommen $\mathrm{zu} \mathrm{N}$ in (11):

(11) zum Abschluss kommen

zur Darstellung kommen

zur Aufführung kommen

zur Versteigerung kommen

zum Ausbruch kommen

zur Anzeige kommen

zum Ausdruck kommen

zur Vollendung kommen

zur Durchführung kommen

zur Anwendung kommen

Das FV drücke, so Fleischers Argumentation, zudem eine relativ abstrakte Bedeutung „Aktionsart“ aus (wobei Fleischer auch die Kausativierung als Aktionsart sieht), und es sei daher ungefähr mit den Konjunktionen und Präpositionen in Phraseoschablonen vergleichbar, da diese ebenfalls keine ,volle' lexikalische (d. h. keine designative) Bedeutung trügen. Aufgrund der Tatsache, dass in der Aktionsartenmarkierung eine ,verallgemeinerbare Bedeutung dieser syntaktischen Struktur“ (Fleischer 1997: 135) gesehen werden könne, die etwa mit der Bedeutung „Intensivierung“ der Struktur N für N vergleichbar sei, klassifiziert er FVG als Phraseoschablonen.

Eine Schwierigkeit dieses Klassifikationsversuchs, die Fleischer (1997: 136) selbst einräumt, besteht darin, dass der strukturelle Rahmen von FVG bei weitem 
nicht so fest ist, wie sonst bei Phraseoschablonen üblich (vgl. auch Feilke 2007: 68). So kann Kausativität mindestens durch die Verben setzen, versetzen und bringen ausgedrückt werden (in Bewegung versetzen, zum Erliegen bringen, in Umlauf setzen, Beispiele nach Fleischer 1997: 137). Im Gegensatz dazu sind die Präpositionen und Konjunktionen bei den oben behandelten Phraseoschablonen nicht variabel (einzig $\mathrm{N}$ für $\mathrm{N}$ und $\mathrm{N}$ um $\mathrm{N}$ stehen einander als Varianten gegenüber, die jedoch weitgehend frei austauschbar zu sein scheinen). Auch wenn man, wie hier in dieser Arbeit, Kausativa wie die eben genannten nicht zu den FVG rechnet, besteht dennoch ein hohes Maß an Variabilität: Es gibt eine große Zahl unterschiedlicher Verben, die in FVG vorkommen - z. B. haben, sein, kommen, bringen, zollen, machen, stellen -, ohne dass dabei klar wäre, ob diese gemeinsam eine einzige Phraseoschablone mit starker Variabilität bilden oder eine Vielzahl von eigenen Phraseoschablonen anzunehmen wären. In beiden Fällen entspricht dies jedenfalls nicht dem sonstigen Bild, das Phraseoschablonen bieten.

Ein weiterer Grund dafür, dass FVG sicherlich keine prototypischen Phraseoschablonen darstellen, ist darin zu sehen, dass nahezu alle Fälle, die von Fleischer als Phraseoschablonen geführt werden, eine im weitesten Sinne expressive Semantik haben: Sie dienen dem Ausdruck von Steigerungen (N für/um N), Intensivierungen (Adj. ist Adj., V und V), von Extremen (ein $\mathrm{N}$ von einem $\mathrm{N}$ ), negativen Bewertungen (Es ist zum Heulen/Davonlaufen usw.) oder dem Ausdruck besonderer Sprechereinstellungen (Der und Lehrer?). Wenn man FVG als Phraseoschablonen ansieht, so fallen diese aufgrund ihrer mangelnden Expressivität somit deutlich aus dem Rahmen.

Trotz der hier vorgebrachten Bedenken gegenüber Fleischers Vorschlag sind grundsätzliche Übereinstimmungen zwischen FVG und Phraseoschablonen nicht zu leugnen: Sowohl bei Phraseoschablonen als auch bei den allermeisten FVG handelt es sich um Verbindungen, die Leerstellen für lexikalisches Material eröffnen. Damit wäre zumindest eine - freilich noch nicht sehr differenzierte Einordnung der FVG erreicht. Offen bleibt dabei, ob FVG zu den beliebig mit lexikalischem Material füllbaren und daher produktiven Schablonen (so Feilke 2007: 67f.) oder nur zu den eingeschränkt füllbaren Strukturen gehören. Dies wäre durch weiterführende Untersuchungen zu klären.

\subsubsection{FVG als Kollokationen}

Neben Fleischers Versuch, FVG den Phraseoschablonen unterzuordnen, gibt es in der Forschung die verbreitete Tendenz, FVG als Kollokationen zu behandeln 
bzw. sie in deren Nähe zu rücken (vgl. Wotjak 1994: 655; Feilke 1996: 146ff.; Lutzeier 1995: 97; Schindler 1996: 26, 124; Proost 2004: 294; Hausmann 2007: 218; Burger 2010: 54, 158; Belica/Perkuhn 2015: 209). ${ }^{72}$ Kollokationen sind nach Hausmann (1985; 2004), der sich mehrfach intensiv mit diesem Themenfeld auseinandergesetzt hat, „typische, spezifische und charakteristische Zweierkombinationen von Wörtern“ (1985: 118). Wesentlich für Kollokationen im Verständnis Hausmanns ist, dass Kollokationen

(a) die präferierte Versprachlichung für einen gegebenen Sachverhalt darstellen,

(b) transparent sind und

(c) ihre Elemente in einer spezifischen Relation zueinander stehen, die mit den Begriffen „Basis“ und „Kollokator“ bezeichnet ist (dazu Näheres unten).

Als Beispiele für Kollokationen, an denen diese Eigenschaften im Weiteren erläutert werden sollen, vgl. die Fälle in (12):

(12) eingefleischter Junggeselle, Der Zorn verraucht, Geld abheben, in Strömen regnen, schwer verletzt, frisch gestrichen, Wutanfall

(vgl. Hausmann 1985: 119; Hausmann 1989: 1010)

Betrachtet man diese Beispiele, wird relativ schnell klar, was mit dem Charakteristikum der ,präferierten Versprachlichung' gemeint ist: eingefleischter Junggeselle, Geld abheben, schwer verletzt, Wutanfall usw. sind ohne Zweifel erste Wahl, wenn nach einem treffenden Ausdruck für den gemeinten Sachverhalt gesucht wird. Ob dies in gleichem Maße allerdings auch für in Strömen regnen, Der Zorn verraucht gilt, sei dahingestellt: Es regnet wie aus Eimern, Es gießt, Es regnet stark und andere sind sicher keine schlechteren, weniger präferierten Ausdrücke, und anstelle von Der Zorn verraucht wäre mindestens auch Der Zorn verfliegt akzeptabel. (Eine statistische Überprüfung der Präferenz verschiedener Versprachlichungen lehnt Hausmann 1985: 124 übrigens explizit ab).

In diesem Zusammenhang ist allerdings auch zu fragen, ob nicht auch Phraseologismen präferierte Kodierungen darstellen: Bezeichnet nicht z. B. schlafende Hunde wecken das Gemeinte - in Duden 11, 622 umständlich paraphrasiert als „unnötig jmds. Aufmerksamkeit erregen und sich damit überflüssige Unan-

72 Eine explizite Trennung der FVG von den Kollokationen wird dagegen bei Steyer (2000: 108), Heine (2006: 53, 76), Helbig (2006: 172f.) vertreten. 
nehmlichkeiten bereiten“ - prägnanter und besser als jede andere Versprachlichung? Ist das Kind mit dem Bade ausschütten gegenüber „mit dem Schlechten zugleich auch das Gute verwerfen“ (so Duden 11,383) nicht die wohl bevorzugte Versprachlichung des betreffenden Sachverhalts? Die Präferenz kollokativer Ausdrücke bei der Versprachlichung von Sachverhalten scheint somit kein Spezifikum von Kollokationen zu sein, sondern ist partiell wohl eine Eigenschaft der Phraseologie allgemein.

Mit dem zweiten der von Hausmann genannten Charakteristika von Kollokationen - ihrer Transparenz - ist offenbar gemeint, dass die Bestandteile von Kollokationen mit ihrer wendungsexternen Bedeutung übereinstimmen und daher als nicht-idiomatisch einzustufen sind. ${ }^{73}$ So sei, um eine illustrative Gegenüberstellung von Pöll (2002: 86) aufzugreifen, span. romper el hielo, das Eis brechen` nicht als Kollokation, sondern als Phraseologismus einzustufen, da hielo ,Eis‘ in dieser Wendung „nicht an eine der regulären Lesarten anbindbar“ sei (ebd.), während span. perder la cabeza ,den Verstand verlieren' aufgrund von cabeza ,Verstand', das in dieser Bedeutung auch in anderen Kontexten auftritt, als Kollokation gelten könne (Gleiches gilt für die deutschen Äquivalente der spanischen Beispiele). Dementsprechend ist in der Literatur teilweise auch explizit davon die Rede, dass Kollokationen nicht idiomatisch seien (vgl. Fix 1974-1976: 60ff.; Wotjak/Heine 2005: 145f.; Heine 2006: 53; Helbig 2006: 172).

Bereits die in (12) genannten Fälle, die von Hausmann immerhin als typisch angesehen werden, sowie das von Pöll angeführte Beispiel zeigen jedoch, dass eine klare Abgrenzung zwischen idiomatisch vs. nicht-idiomatisch schwer zu erreichen ist. Ist in Strömen in der Kollokation in Strömen regnen tatsächlich identisch mit der wendungsexternen Verwendung? Liegt hier nicht vielmehr eine bildliche Verwendung vor ,es regnet so stark wie ein Strom, der gewissermaßen von oben kommt'? Die reguläre Lesart von Strom ist hier wohl kaum gegeben. Span. perder la cabeza bzw. dt. den Verstand verlieren sind entgegen der Auffassung Pölls ebenfalls nicht uneingeschränkt transparent: Zwar entsprechen cabeza bzw. Verstand der wendungsexternen Bedeutung, aber sowohl perder als auch verlieren zeigen nicht die erwartbare Lesart, da das Objekt von perder/verlieren normalerweise nur ein konkreter Gegenstand, keine abstrakte Entität ist. Der gleiche Einwand gilt mindestens auch für die angebliche Kollokation schwer verletzt, da schwer (im Sinne von ,stark, sehr') sicher nicht der prototypischen Lesart des Adjektivs entspricht, die wohl eher in eine schwere Einkaufstasche, der

$73 \mathrm{Zu}$ einer ersten Bestimmung des Idiom-Begriffs vgl. Burger (2010: 30), der Idiomatizität als „Diskrepanz zwischen der phraseologischen und der wörtlichen Bedeutung des ganzen Ausdrucks" beschreibt. 
schwere Mann u. ä. vorliegt. Daher ist Hausmanns Auffassung, Kollokationen seien prinzipiell transparent bzw. nicht-idiomatisch, da ihre Bestandteile ungebrochen den wendungsexternen Bedeutungen entsprechen, in dieser Zuspitzung nicht zu halten. Man wird den Verhältnissen hier wohl eher gerecht, wenn man Kollokationen in nicht-idiomatische und schwach idiomatische unterteilt (mit Übergängen zwischen diesen Gruppen; vgl. auch Burger 2010: 52). Dabei kann man die nicht-idiomatischen Kollokationen durchaus als die prototypischeren ansehen, da sie am deutlichsten einen Gegenpol zu den Phraseologismen bilden, die sich typischerweise durch hohe Idiomatizität auszeichnen.

Im Vergleich mit den bisher diskutierten Kriterien erscheint die letzte der eingangs genannten Eigenschaften von Kollokationen - die besondere Relation zwischen den beteiligten Elementen - noch am besten fassbar. Das Verhältnis zwischen den in einer Kollokation verbundenen Lexemen ist Hausmann zufolge so zu beschreiben, dass ein Lexem die Basis, das andere den Kollokator bildet: Der Basisbestandteil weist eine autonome Lesart auf, die auch außerhalb der Verbindung gilt, während der zweite Bestandteil, der sog. Kollokator, seine Lesart nur in Abhängigkeit von der Basiskomponente erhält. So wäre z. B. Geld als die Basis zu betrachten, abheben als Kollokator, da nur das Vorkommen mit Geld festlegt, dass für das Verb genau die vorliegende Lesart gilt und etwa nicht die in Karten abheben greifbare Bedeutung. Durch das Gegebensein einer Basis-Kollokator-Relation sind Kollokationen von den Phraseologismen gut unterscheidbar: für einen typischen Phraseologismus wie auf dem Trockenen sitzen ist eine solche Relation nicht zu beschreiben, da hier unbestimmt bleibt, ob das Verb die PP oder die PP das Verb hinsichtlich der semantischen Interpretation determiniert. ${ }^{74}$

74 Zwar stellt die Relation Basis-Kollokator ein auf den ersten Blick praktikables Instrument für die Ermittlung von Kollokationen dar. Dennoch birgt dieses Konzept nicht unerhebliche Schwierigkeiten, auf die hier allerdings nur andeutungsweise eingegangen werden kann. Dass in der Verbindung Geld abheben das Nomen Geld die Lesart des Verbs abheben determiniert, erscheint nicht weiter verwunderlich, wenn man bedenkt, dass Geld ohnehin nur eine Lesart, nämlich ,Zahlungsmittel', aufweist, während abheben mindestens, anheben und entfernen ' (den Deckel abheben), ,sich in die Luft erheben' sowie ,optisch hervortreten lassen' bedeuten kann (zum gesamten Bedeutungsspektrum s. DUW 82). Dass das tendenziell monoseme Wort quasi die Bedeutungen des polysemen Wortes selegiert, erscheint kaum anders denkbar und bedarf nicht eigens eingeführter Begriffe wie „Basis“ und „Kollokator“. Betrachtet man anstelle von Geld abheben das gleichbedeutende umgangssprachliche Kohle abheben, kann die Frage, welches Element Basis und welches Kollokator ist, nicht mehr so leicht beantwortet werden: Determiniert Kohle tatsächlich die Lesart von abheben? Kann man nicht auch sagen, dass abheben die Lesart von Kohle determiniert, da dieses ja ebenfalls mehrdeutig ist? Vergleichbare Fragen lassen sich auch an zentrale Beispiele für Kollokationen richten: In frisch gestrichen wäre Hausmann (1985) zufolge 
Wenn die Forschung zur Phraseologie FVG zumeist den Kollokationen zuordnet, so geschieht dies weitgehend ohne Begründung oder Diskussion. Eine der wenigen ausführlicheren Erörterungen dieser Frage findet sich bei Helbig (2006: 172f.). Helbig sieht vor allem in der Nicht-Idiomatizität der Bestandteile eine gemeinsame Eigenschaft von Kollokationen und FVG (in diesem Sinne auch Roos 2001: 16; Wotjak/Heine 2005: 145; Heine 2006: 54, 203). Dass Verben wie kommen, bringen, geben, stellen, treffen usw. in FVG nicht mit den üblichen Bedeutungen dieser Verben (die ja primär Bewegungs-, Positions- oder Transferverben sind) übereinstimmen, liegt indes auf der Hand, schließlich sind diese Verben, wenn sie als FV auftreten, lexikalisch-semantisch leer. Es hilft auch wenig, die semantische Abweichung der Verben in FVG als „,semantic specialization“ (Burger 2007: 91) zu beschreiben und mittels eines solchen terminologischen Handgriffs der eigentlich naheliegenden Feststellung, dass hier Idiomatizität vorliegt, auszuweichen, zumal das gänzliche Fehlen lexikalisch-semantischer Merkmale nur schwer als „specialization“ einer Vollverblesart bezeichnet werden kann. So stellt auch Fleischer (1997: 35) mit Recht fest, dass es sich bei den FVG (illustriert am Beispiel der Fügungen mit treffen) „trotz der Reihenbildung um eine ,irreguläre“ Kombination in Bezug auf die Semantik von treffen“ und demnach „um teilidiomatische Phraseologismen“ handelt. (Von ,teilidiomatisch“ ist hier bei Fleischer deshalb die Rede, weil das Verb idiomatisch ist, die NP/PP hingegen nicht). Die Teilidiomatizität von FVG hebt diese somit deutlich von den Kollokationen ab, die zumindest nach Hausmanns Verständnis als transparent, d. h. als grundsätzlich nicht idiomatisch zu bewerten sind, vgl. sein Kriterium (b). ${ }^{75}$

\footnotetext{
gestrichen die Basis, frisch der Kollokator, da gestrichen bestimmt, dass frisch die Lesart, vor kurzer Zeit' o. ä. erhält. Könnte man hier nicht mit gleichen Recht behaupten, dass frisch die Lesart von gestrichen bestimmt, da letzteres ebenfalls mehrdeutig ist (streichen, wegfallen lassen', ,mit einer gleitenden Bewegung über etwas hinfahren' usw.)? So ließe sich postulieren, dass frisch ,vor kurzer Zeit‘ alle anderen Lesarten von streichen ausschließt und nur die Lesart ,mit Farbe anstreichen' selegiert. Vielleicht erscheint es angesichts der Schwierigkeit, diese Fragen zu beantworten, angebrachter, die Redeweise von Wörtern, die Lesarten anderer Wörter selegieren oder determinieren, grundsätzlich zu aufzugeben. Anstelle einer solchen Metapher, die Wörtern Fähigkeiten zuschreibt, die normalerweise nur Sprecher bzw. Hörer haben, wäre es möglicherweise realistischer zu sagen, dass im Wesentlichen der außersprachliche Kontext, in dem eine Äußerung produziert bzw. rezipiert wird, die Lesarten von Wörtern determiniert: Dass die Wörter in frisch gestrichen genau diese und keine anderen Bedeutungen aufweisen, ergibt sich in erster Linie aus dem für Sprecher und Hörer gegenwärtigen Kontextbezug.

75 Auch Helbig (2006: 171f.) kommt letztlich zu dem Schluss, dass FVG nicht als Teilmenge der Kollokationen zu gelten hätten, sondern als eigene Subklasse der Phraseologismen zu beschreiben seien. Seine Argumentation stützt sich dabei aber z. B. auf die Beobachtung, dass FVG ein gemeinsames Prädikat bildeten und z. B. Angst in Angst haben daher kein Akkusativobjekt sei;
} 
Auch Lutzeier (1995: 97f.) hat sich ausführlicher mit der Frage nach dem genauen Verhältnis von FVG zu Kollokationen auseinandergesetzt. Lutzeier stützt sich in seiner Argumentation auf das Basis-Kollokator-Verhältnis (Kriterium c): FVG seien, so Lutzeier, dahingehend zu deuten, dass die Verben erst durch die Kookkurrenz mit ihren Substantiven die einschlägige Lesart zugewiesen bekommen. So bemerkt Lutzeier (1995: 97) zu fällt eine Entscheidung: „Die Nominalphrase eine entscheidung [sic] erhält natürlich ihre gewöhnliche Interpretation, während die Form fällt ihre Lesart in entscheidender Weise über die Nominalphrase eine entscheidung erhält".

So zutreffend diese Beschreibung ist, muss man gleichwohl festhalten, dass eine Basis-Kollokator-Relation, anders als Hausmann annimmt, durchaus nicht nur bei Kollokationen auftritt. Wenn man das Verhältnis zwischen Basis und Kollokator in der Weise bestimmt, dass die Basis das Element einer Wortverbindung ist, das die Lesart des zweiten Elements, des Kollokators, determiniert, dann kann auch für Phraseologismen wie einen Streit vom Zaun brechen eine Basis-Kollokator-Relation angesetzt werden. Durch das Vorkommen des Nomens Streit ist festgelegt, dass die VP vom Zaun brechen nicht wörtlich im Sinne von, etwas (z. B. eine Latte) vom Zaun abbrechen' $z u$ interpretieren ist, sondern dass die Bedeutung ,anfangen' o. ä. vorliegt. Die VP vom Zaun brechen erhält ihre Lesart somit über die NP Streit, wie eben abheben eine Lesart von der NP Geld zugewiesen wird (um eine unstrittige Kollokation zu nennen). Nun gelten Fügungen wie einen Streit vom Zaun brechen traditionellerweise gerade nicht als Kollokationen, sondern als teilidomatische Phraseologismen (vgl. Burger 2010: 30; weitere Beispiele für teilidiomatische Phraseologismen wären jmdm. ein Loch in den Bauch fragen, die Zeit totschlagen oder aus vollem Halse lachen). Die Basis-Kollokator-Relation ist, da sie nicht nur FVG, sondern auch teilidiomatische Phraseologismen auszeichnet, zu unspezifisch, als dass sie eine Subsumierung der FVG unter die Kollokationen rechtfertigen könnte.

Das Kriterium der präferierten Versprachlichung (Kriterium a), das für Hausmanns Kollokationsbegriff wesentlich ist, ist ebenfalls nicht geeignet, eine Unterordnung der FVG unter die Kollokationen plausibel zu machen. Fügungen wie Antwort geben, Anwendung finden, zur Aufführung kommen, sind, anders als die

Kollokationen wie Geld abheben besäßen hingegen eine reguläre syntaktische Struktur. Auch wenn man dem zustimmt, wäre allein damit nicht ausgeschlossen, dass es sich bei FVG um eine Subklasse der Kollokationen handelt. Relevant für diese Frage ist allein, ob FVG die wesentlichen Eigenschaften, die Kollokationen ausmachen, teilen oder nicht. Dafür ist eine Liste ,harter Kriterien für Kollokationen aufzustellen, wie es z. B. mit dem oben diskutierten Hausmann'schen Merkmalkatalog vorliegt. ,Reguläre‘ vs. ,nicht-reguläre syntaktische Struktur‘ wird aber auch von Helbig (2006) nicht als generelles Unterscheidungskriterium für Kollokationen postuliert. 
in (12) genannten Beispiele für Kollokationen, nicht die erste Wahl bei der Versprachlichung des betreffenden Sachverhalts. Präferiert sind hier grundsätzlich die einfachen Verbalausdrücke antworten, angewandt werden, aufgeführt werden. ${ }^{76}$

Entschieden gegen eine enge Verbindung zwischen Kollokationen und FVG spricht aber vor allem der Bedeutungsaufbau der betreffenden Fügungen. Das Kriterium des Bedeutungsaufbaus spielt für die von Hausmanns gebotene Definition des Kollokationsbegriffs zwar keine Rolle, gleichwohl ist damit ein deutlicher Unterschied zwischen FVG und Kollokationen markiert. Kollokationen zeichnen sich grundsätzlich dadurch aus, dass sie semantisch teilbar sind: Geld abheben ist in zwei Komponenten zerlegbar, denen eine eigenständige lexikalische Bedeutung zugewiesen werden kann (zur semantischen Teilbarkeit von Phraseologismen s. auch weiter Abschnitt 4.2.5). Kollokationen verhalten sich in dieser Hinsicht wie Einheiten der freien Syntax. Bei FVG ist hingegen keine semantische Teilbarkeit gegeben - zumindest wenn man der in Kapitel 2 vorgeschlagenen Definition folgt: Dem Nomen kann zwar eine lexikalische Bedeutung zugewiesen werden, für das Verb ist das jedoch nicht der Fall. Kollokationen und FVG wird man daher auseinanderhalten müssen.

\subsubsection{FVG als Enkodierungsidiome}

Auch wenn Hausmanns Kollokationsbegriff nicht auf FVG anwendbar ist, bleibt dennoch festzuhalten, dass FVG durchaus keine frei variierbaren Nomen-VerbVerbindungen sind. Die Möglichkeit, Nomina und Verben beliebig miteinander zu kombinieren, besteht nicht (vgl. Feilke 2007: 68). So können die NPs und PPs in den Verbindungen in (13) nur mit einem oder zwei Verben verbunden werden, wenn die FVG-Lesart erhalten bleiben soll. Hier sind zum Teil sogar Verben ausgeschlossen, die derselben semantischen Klasse angehören wie die in FVG akzeptablen Verben: ${ }^{77}$
a. zur Aufführung kommen/gelangen/\#ankommen
b. zur Aufführung bringen/\#transportieren
c. Antwort geben/erteilen/^austeilen/\#übergeben

$76 \mathrm{Zu}$ der auch in Korpora unmittelbar aufscheinenden wesentlichen höheren Frequenz einfacher Verben gegenüber den parallelen FVG vgl. Storrer (2006: 159).

$77 \mathrm{Zu}$ dieser Beobachtung vgl. auch Krenn/Erbach (1994: 379f.). 

d. Anwendung finden/*auffinden/\#entdecken
e. einen Besuch machen/?tun

Umgekehrt sind auch die Substantive vielfach nicht durch bedeutungsnahe Alternativen zu ersetzen:
a. zur Aufführung/Uraufführung/`Premiere/*Generalprobe kommen/ bringen
b. einen Beschluss fassen/*eine Entscheidung fassen
c. zur Versteigerung/'Auktion kommen

Die Möglichkeiten, ein FVG mit bringen/kommen zu bilden, sind folglich - so scheint es zumindest auf der Basis der Beispiele in (14) - nicht vorhersagbar: Bei Beschluss ist eine Kombination mit fassen möglich, bei Entscheidung nicht. Aufgrund dieser Arbitrarität können FVG zu den sog. ,encoding idioms“ gerechnet werden (vgl. Makkai 1972: 25; Fillmore/Kay/O’Connor 1988: 504f.). Bei Enkodierungsidiomen im Sinne von Fillmore et al. handelt es sich um Verbindungen, die nicht frei gebildet, d. h. nicht frei ,enkodiert' werden können, die aber auf der Basis genereller Interpretationsregeln für Äußerungen durchaus verstehbar sind, also problemlos , dekodiert‘ werden können.

Als Beispiele für Enkodierungsidiome werden die englischen Ausdrücke answer the door ,die Tür auf Klopfen hin öffnen', wide awake, vollständig wach“ und bright red ,knallrot' genannt. Für diese gilt:

[W]hile it is likely that each expression of the latter group [Enkodierungsidiome, V. H.] could be understood perfectly on first hearing, someone who did not know that they were conventional ways of saying what they say would not be able to predict their usability in these ways.

(Fillmore/Kay/O’Connor 1988: 505)

Enkodierungsidiome sind nur in der Perspektive des Sprechers als idiomatisch anzusehen. Feilke (2004: 55, 60) spricht daher zutreffend auch von „Produktionsidiomen“. Aus der Sicht des Hörers stellen sich diese Idiome nicht als problematisch dar, da deren Bedeutung sich von selbst erschließt, wenn die einzelnen Wortbedeutungen bekannt und generelle metaphorische und metonymische Interpretationstechniken verfügbar sind (wie z. B. Grad der Wachheit korrespondiert mit Öffnungsgrad der Augen usw. im eben genannten Beispiel engl. wide awake).

Von den Enkodierungsidiomen sind nach Fillmore/Kay/O'Connor (1988: 504f.) die Dekodierungsidiome zu unterscheiden. Diese müssen nicht nur seitens 
des Sprechers gelernt werden, sondern auch ein Hörer muss lernen, deren Bedeutung zu entschlüsseln, da sie sich nicht aus den Teilbedeutungen ergibt. So ist die Wendung engl. kick the bucket ,sterben' (Beispiel Fillmore/Kay/O’Connor 1988: 505) auf der Basis ihrer Teilbedeutungen sowie allgemeiner Interpretationsregeln weder verstehbar noch frei bildbar. Sowohl die Dekodierung als auch die Enkodierung muss in diesem Fall gelernt werden. Alle Dekodierungsidiome sind daher grundsätzlich auch Enkodierungsidiome (aber nicht umgekehrt).

Der Begriff des Enkodierungsidioms weist, wie unschwer zu erkennen, Gemeinsamkeiten mit dem der Kollokation auf, da es in beiden Fällen um „,conventional ways of saying“ geht (Fillmore/Kay/O’Connor 1988: 505; vgl. auch Croft/Cruse 2004: 250). Freilich ist der Begriff ,Enkodierungsidiom“ noch weniger scharf gefasst als ,Kollokation“, so dass viele Fragen offen bleiben. Insbesondere ist unklar, ob nicht-idiomatische Kollokationen wie frisch gestrichen ebenfalls als Enkodierungsidiome zu führen sind; die Beispiele, die Fillmore et al. nennen, lassen eher darauf schließen, dass es sich bei den „encoding idioms“ um stärker idiomatisierte Bildungen handelt, als bei der Kollokationen der Fall ist. Aufgrund der Unklarheit des Begriffs ist es schwer, das Verhältnis der FVG zu den Enkodierungsidiomen zu bestimmen. Tendenziell wird man sie aber eher den Enkodierungsidiomen als den Dekodierungsidiomen zuschlagen, da die Schwierigkeiten ihrer Interpretation beherrschbar sind: Dass ein FVG wie zur Aufführung kommen ,aufgeführt werden' bedeutet, erfordert deutlich weniger interpretatorischen Aufwand, als wenn z. B. die Bedeutung der Phrase wissen, wo Barthel den Most holt (eines typischen Dekodierungsidioms) auf der Basis des Äußerungskontextes erschlossen werden muss.

Das Konzept des Enkodierungsidioms ist aber in unserem Zusammenhang insofern von Nutzen, als es hilft, eine grundlegende Frage etwas genauer zu formulieren: Muss die Enkodierbarkeit jedes einzelnen FVG gelernt werden, muss also gelernt werden, dass zur Aufführung bringen bildbar ist, *zur Premiere bringen hingegen kein „,conventional way of saying“ ist? Oder gibt es möglicherweise eher ein wie auch immer geartetes allgemeines Muster PP + bringen, das gespeichert ist und dessen PP-Position relativ frei gefüllt werden kann? Nach Ausweis der Beispiele in (14) sieht es hier vorläufig so aus, dass kein Muster vorliegt und tatsächlich jede Bildung einzeln erlernt und abgespeichert wird. Dies ist freilich noch im Einzelnen zu prüfen (s. Kapitel 5).

Die von Fillmore/Kay/O’Connor (1988) entwickelte Klassifikation der Idiome - in der deutschsprachigen Tradition würde man eher von ,Phraseologismen' sprechen - enthält eine weitere Unterscheidung, die für die Klassifikation von FVG nützlich sein kann, nämlich die Differenzierung nach der Vertrautheit 
der Komponenten eines Phraseologismus sowie der Art ihrer Kombination. Dementsprechend unterscheiden Fillmore/Kay/O’Connor (1988: 506-510) zwischen:

- „unfamiliar pieces unfamiliarily arranged“ (z. B. engl. kith and kin, dt. gang und gäbe),

- „familiar pieces unfamiliarily arranged“ (z. B. engl. all of a sudden, in point of fact, dt. Kunst muss nach Brot gehen),

- „familiar pieces familiarily arranged” (engl. to pull someone’s leg; dt. sich die Kante geben).

Einschlägig für FVG dürfte das zweite dieser Kombinationsmuster sein, die „familiar pieces unfamiliarily arranged”, da FVG - bis auf wenige Ausnahmen wie die Bildungen mit zollen - keine unikalen Lexeme (,unfamiliar pieces“) enthalten, die Verbindung dieser Lexeme hinsichtlich des Bedeutungsaufbaus aber ungewöhnlich ist, wie in Kapitel 3.2.2 gezeigt wurde. Das Kombinationsmuster „familiar pieces unfamiliarily arranged” wird wie folgt erläutert: „Here [...] the semantic interpretation is necessarily a novel one, since the principles of combination used for general semantic interpretation cannot serve us here“ (ebd. 508). Wenn die generellen Interpretationsprinzipien hier nicht gelten, so können nach Fillmore et al. allerdings, sofern es sich um Phraseoschablonen/,formal idioms“ handelt, spezielle Regeln für die betreffenden Kombinationen formuliert werden: „[...] the internal syntax and semantics of such phrases require a special minigrammar embedded within the general grammar, whose properties are not deducible from those of the larger grammar“ (ebd. 510). Das System der FVG - wenn diese denn als System beschreibbar sind - wäre durchaus ein Kandidat für eine solche ,Mini-Grammatik' mit begrenzter Geltung. Welchen Regeln eine solche Mini-Grammatik möglicherweise gehorcht, wird in den folgenden Kapiteln erörtert.

\subsubsection{FVG und die Teilbarkeit von Phraseologismen}

Die Position der FVG innerhalb der phraseologischen Erscheinungen lässt sich möglicherweise leichter bestimmen, wenn man auf vorgefertigte Kategorien wie etwa ,Kollokation“ oder ,Phraseoschablone“ verzichtet und stattdessen empirisch besser überprüfbare Kriterien heranzieht. Als ein Kriterium, das die Stellung einer Fügung innerhalb der Phraseologie verdeutlichen kann, wird gelegentlich das Fehlen bzw. Vorhandensein semantischer Dekomponierbarkeit genannt (vgl. Abschnitt 4.2.3): Phraseologismen, die sich nicht in bedeutungshaltige Komponenten teilen lassen, werden als zentral angesehen, während teilbare Fügungen 
als peripher betrachtet werden, da Dekomponierbarkeit eine grundlegende Eigenschaft regulärer Verbindungen ist (Dobrovol'skij 1995: 29; vgl. Cruse 2011: 86). Als semantisch teilbar gelten Wendungen wie jmdm. einen Bären aufbinden, nicht teilbar sind demgegenüber Phraseologismen wie ins Gras beißen. Begründet wird diese Unterscheidung damit, dass im Fall der zuerst genannten Fügung eine Parallelität zwischen einzelnen Komponenten des Phraseologismus und den Komponenten der Bedeutungsparaphrase konstruiert werden kann, wie (15) zeigt; bei (16) liegt hingegen keine derartige Parallelstruktur vor.

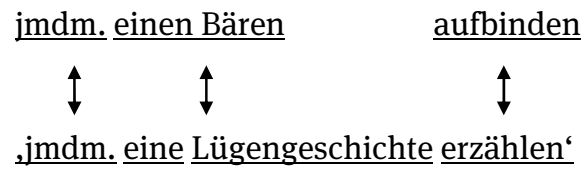

(16) ins Gras beißen

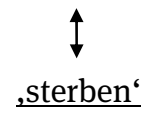

Ein dekomponierbarer Phraseologismus wie jmdm. einen Bären aufbinden zeichnet sich dadurch aus, dass allen Teilen eine - freilich nur verbindungsintern gültige - Bedeutung zugeschrieben werden kann; bei nicht dekomponierbaren Verbindungen wie ins Gras beißen kann hingegen für keines der Elemente eine Bedeutung konstruiert werden, und sei diese nur wendungsintern. Hier gibt es nur eine globale Bedeutung.

FVG verhalten sich in dieser Hinsicht jedoch paradox: Sie sind insofern mit dem Typ ins Gras beißen ,sterben“ vergleichbar, als nicht jedem Element der Paraphrase ein einzelnes Ausdruckselement zugeordnet werden kann. Wenn man sich darauf verständigt, dass zur Aufführung bringen ,aufführen“ bedeutet, dann entspricht dies den Verhältnissen bei ins Gras beißen ,sterben', da hier wie dort zwei syntaktische Einheiten - PP und V - mit einer globalen Bedeutungsangabe korrespondieren. Gleichwohl wäre es unangemessen, ein FVG wie zur Aufführung bringen schlicht zu den nicht-kompositionellen Phraseologismen zu zählen. Es besteht zwar keine Parallelität zwischen dem Inhalt und den syntaktischen Komponenten, gleichwohl ist zur Aufführung bringen im Unterschied zu ins Gras beißen durchsichtig: Vom Nomen ausgehend kann die lexikalische Bedeutung der gesamten Fügung erschlossen werden. Das Verb steuert lediglich die Information ,aktiv‘ bei (s. o. Kapitel 3.2.4). Bei anderen FVG wie zur Aufführung kommen ,aufgeführt werden' oder Berücksichtigung finden ,berücksichtigt werden' ist es noch schwerer, überhaupt einen semantischen Beitrag des Verbs zu identifizieren: Die 
Substantive Aufführung und Berücksichtigung sind für sich genommen bereits passivisch zu interpretieren (s. Kapitel 3.2.4), daher drückt kommen bzw. finden in lexikalisch-semantischer Hinsicht gar nichts aus.

FVG nehmen damit eine merkwürdige Position ein: Sie sind nicht dekomponierbar wie Phraseologismen des Typs jmdm. einen Bären aufbinden, weil keine Isomorphie zwischen den syntaktischen Einheiten und der natürlichsprachigen Bedeutungsparaphrase der Verbindung vorliegt. Sie sind aber auch nicht kompositional wie ein reguläres Syntagma des Typs ein Buch lesen, ${ }^{78}$ da das Verb semantisch leer ist. Die Gesamtbedeutung eines FVG kann somit nicht in der üblichen Weise aus den Bedeutungen der Teile zusammengesetzt werden. Aus dieser Beobachtung ergibt sich immerhin die Möglichkeit, für FVG eine eigene Form der Kompositionalität nach dem Schema „1+0 = 1“ postulieren. Dies gälte zumindest für die tautologischen Fügungen, die in der hier zugrundegelegten Klassifikation immerhin den Kern der Kategorie ,FVG‘ bilden. FVG könnten damit in der Tat im Sinne von Fillmore/Kay/O’Connor (1988: 510) als „,special mini-grammar embedded within the larger grammar“, als Subbereich der Grammatik beschrieben werden, in dem eine spezielle Regel gilt.

Ein weiterer Aspekt macht die Angelegenheit freilich noch komplexer. Die Antwort auf die Frage, ob ein Phraseologismus dekomponierbar ist, hängt entscheidend von den jeweils gewählten Paraphrasen ab. Die Kompositionalität vs. Nicht-Kompositionalität eines Phraseologismus ist damit, so Dobrovol'skij (1995: 23), „kein ontologisches, sondern eher ein technisches Kriterium“. Wenn man also anstelle eines normalsprachlichen Beschreibungsinventars die formalisierten Bedeutungsbeschreibungen der lexikalischen Semantik mit Prädikaten wie CAUSE, DO, BECOME etc. zugrunde legt, ergibt sich möglicherweise ein vollkommen anderes Bild: Ins Gras beißen korrespondiert dann nicht mit ,sterben', sondern mit [BECOME $(\mathrm{x}<D E A D>)$ ] (in diese Richtung geht z. B. der Vorschlag von G. Müller 2011: 213f.). Mit Hilfe einer solchen Dekomposition könnte sehr wohl eine Parallelität zwischen Phraseologismus und Bedeutungsparaphrase konstruiert werden: Das BECOME-Prädikat wäre dann mit dem Verb beißen und die PP ins Gras mit der Bedeutungskomponente DEAD in Beziehung zu setzen, s. (17).

$$
\begin{aligned}
& \underline{\text { beißt }} \underline{\text { ins Gras }} \\
& {[\operatorname{BECOME}(\mathrm{x}<D E A D>)]}
\end{aligned}
$$

78 Inwiefern die Intuition zutrifft, dass ein Buch lesen vollkommen regulär bzw. kompositionell, zutrifft, wird von Sailer (2003) ausführlich dargelegt (am Beispiel von engl. to read a book). 
Allerdings ist eine solche Analyse mit problematischen Zusatzannahmen verbunden: Der PP ins Gras wird ein semantischer Gehalt zugeschrieben, ohne dass dies in irgendeiner Weise testbar wäre. Dass dagegen einen Bären in (15) eine wendungsintern gültige Bedeutung besitzt, zeigt sich daran, dass hier Attribuierung möglich ist (jmdm. einen dicken Bären aufbinden). Die Gleichsetzung von ins Gras mit einer Komponente ( $\mathrm{x}<\mathrm{DEAD}>$ ) bleibt demgegenüber somit eine Stipulation.

Trotz der Bedenken gegenüber einer Analyse wie in (17) ist auch für FVG zu prüfen, ob sich bei Zugrundelegung einer formalen semantischen Beschreibungssprache Differenzierungen gegenüber dem bisherigen Befund ergeben, dass FVG nicht kompositional im üblichen Sinne sind. Legt man auch für FVG Paraphrasen beispielsweise im Stile der von Levin/Rappaport Hovav (1998: 108) entwickelten semantischen Beschreibungssprache zugrunde, wäre die Bedeutung von zur Aufführung kommen, wie die von aufgeführt werden, mit der Notation in (18) wiederzugeben (hier nach dem Muster der Notation für Achievements). Hier könnte man dann eine Entsprechung zwischen kommen und zur Aufführung auf der einen und einzelnen Komponenten der Bedeutungsbeschreibung auf der anderen Seite konstruieren.

\section{(18) kommt zur Aufführung \\ [BECOME $(\mathrm{y}<A U F G E F U ̈ H R T>)]$}

Auf andere FVG wie z. B. das ebenfalls tautologische Antwort geben ist diese Analyse freilich nicht übertragbar. Antwort geben wäre wie antworten grundsätzlich als Activity zu klassifizieren; es müsste Levin/Rappaport Hovav (1998: 108) folgend als einfaches Do-Prädikat notiert werden. Damit ergibt sich aber, wie (19) zeigt, ein gänzlich anderes Verhältnis zwischen den Ausdrucks- und Inhaltskomponenten als in (18).

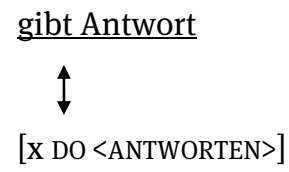

Da die FVG Antwort geben und zur Aufführung kommen hinsichtlich ihres Bedeutungsaufbaus zu einer identischen Klasse, nämlich zu den tautologischen Verbindungen, gehören, sind die unterschiedlichen Form-Inhalt-Beziehungen, die sich aus (18) und (19) ergeben würden, wenig plausibel. Bereits diese beiden Bei- 
spiele belegen also, dass für FVG auch dann, wenn man eine formalere Beschreibungssprache zugrunde legt, kein kompositionaler Bedeutungsaufbau nachweisbar ist.

Im Hinblick auf das Kriterium der Kompositionalität bzw. Zerlegbarkeit erweisen sich FVG somit insgesamt als inkommensurabel: Einerseits sind sie semantisch nicht zerlegbar und entsprechen damit einem prototypischen Phraseologimus wie ins Gras beißen. Andererseits unterscheiden sie sich von Phraseologismen wie dem genannten darin, dass sie mit dem Nomen in der NP/PP eine zentrale Komponente aufweisen, die der verbindungsexternen Bedeutung vollkommen entspricht. Hier liegt somit das paradoxe Phänomen einer nicht zerlegbaren daher eigentlich stark idiomatischen Verbindung vor, die aber semantisch sehr transparent und daher eigentlich nicht idiomatisch ist. Unter dem Gesichtspunkt der Zerlegbarkeit bilden FVG somit in der Tat eine Kategorie zwischen Phraseologie auf der einen und regulärer Syntax auf der anderen Seite.

\subsection{FVG als Konstruktionen}

\subsubsection{Vorüberlegungen}

Da FVG sich bisher weder als eindeutig grammatische noch als eindeutig phraseologische Erscheinungen erwiesen haben, bleibt deren Position innerhalb des Sprachsystems immer noch unklar. Zwar hat die Auffassung, dass FVG in einem „Grenzbereich der Phraseologie zur Syntax“ (Fleischer 1997: 131) anzusiedeln seien - „[s]omewhere near the middle of the magnetic field of language [...], where grammar and lexis meet“ (Algeo 1995: 203) -, einiges für sich (vgl. auch Detges 1996: 4; Busse 2002: 412; van Pottelberge 2007: 436). Allerdings ist eine solche Standortbestimmung mit zwei sehr grundsätzlichen Problemen verbunden: Zum einen ist, wie in Kapitel 2 gezeigt worden ist, der Terminus „FVG“ in der bisherigen Forschung zu schlecht bestimmt, als dass eine Einordnung der Kategorie in einen größeren Zusammenhang Aussichten auf Erfolg haben könnte erst wenn bekannt ist, was genau einzuordnen ist, kann eine Positionsbestimmung überhaupt gelingen. Zum anderen hängt die Aussage, FVG gehörten einem Grenzbereich zwischen Phraseologie und Syntax an, in sprachtheoretischer Hinsicht gewissermaßen in der Luft, da in den gängigen sprachwissenschaftlichen Beschreibungsmodellen ein Übergangsbereich zwischen Syntax und Phraseologie nicht vorgesehen ist. So wird in der Tradition der generativen Grammatik überwiegend eine strikte Trennung von Syntax und Lexikon (zu dem auch die Phraseologie gehört) vertreten. Eine solche strikt modulare Auffassung von Spra- 
che sieht allenfalls spezifische Schnittstellen zwischen den einzelnen Subsystemen der Sprache vor; Übergangszonen wie der von Fleischer und anderen postulierte „Grenzbereich zwischen Syntax und Phraseologie“ werden aber nicht angenommen. Wenn der von Fleischer behauptete Bereich zwischen Phraseologie und Syntax nicht bloß ein ad-hoc-Postulat sein soll, ist eine theoretische Rückbindung dieser Aussage erforderlich.

Der ersten Schwierigkeit, die sich allen Einordnungsversuchen stellt - der präzisen Bestimmung dessen, was überhaupt klassifiziert werden soll -, ist mit der in Kapitel 3 vorgeschlagenen Definition des FVG als Syntagma mit implikativem Bedeutungsaufbau zumindest insoweit abgeholfen, als dass hierdurch eine Verortung der betreffenden Fügungen eher möglich sein sollte als auf der Basis der bisherigen, weitestgehend unscharf bleibenden Begriffsbestimmungen. Das zweite oben angesprochene Problem - der sprachtheoretische Rahmen, auf den sich eine Einordnung der FVG zwischen Syntax und Phraseologie überhaupt beziehen kann - bedarf allerdings noch einer Lösung. Diese ist nur in einem Beschreibungsmodell möglich, das die Sprache nicht in abgeschlossene Module einteilt, sondern Übergangszonen zwischen Teilsystemen zulässt. Ferner sollte dieses Modell auch einen hohen Auflösungsgrad aufweisen, da viele kleine, ggf. skalenartig gestufte Kategorien eine bessere Einordnung einzelner Erscheinungen wie der FVG erlauben als Modelle, die nur relativ große Blöcke wie „Syntax“, „Basiskomponente“, „Logische Form“ o. ä. unterscheiden.

Ein Sprachmodell, das die eben genannten Anforderungen erfüllt, stellt die sog. Konstruktionsgrammatik dar. Anstelle des modularen Ansatzes der generativen Modelle geht die Konstruktionsgrammatik von einem Lexikon-Syntax-Kontinuum aus, das mehrere Übergangsstufen zwischen den beiden Polen Lexikon und Syntax enthält. Ein feinkörniges Modell wie dieses lässt durchaus erwarten, das eine Erscheinung, die sich in die traditionelle Dichotomie Syntax vs. Lexikon (inklusive Phraseologie) nicht einfügen will, adäquat beschrieben werden kann.

Bevor eine konstruktionsgrammatische Analyse von FVG in Angriff genommen werden kann, sind im Folgenden einige Grundzüge dieser Theorietradition darzulegen, zumal auch in Kapitel 7 auf Positionen der Konstruktionsgrammatik zurückzukommen ist.

\subsubsection{Grundzüge der Konstruktionsgrammatik}

In der traditionellen Grammatik werden Kategorien wie ,Nomen', ,Präposition', ,Passiv‘ u. ä. im Allgemeinen als Verbindungen von Form und Funktion - als 
grammatische Konstruktionen - aufgefasst. Die Überzeugung von einem semantischen Gehalt und damit von der Zeichenhaftigkeit grammatischer Kategorien schlägt sich besonders deutlich in - allerdings längst außer Gebrauch gekommenen - Bezeichnungen wie „Dingwort“ oder „Zeitwort“ nieder (ein ähnliches Verständnis liegt bekanntermaßen auch zahlreichen griechisch-römischen Termini, die die Vorlage vieler dieser Bildungen sind, zugrunde): So ist z. B. ein „Dingwort“ bzw. Substantiv nicht einfach nur ein Wort, das flektierbar ist, sondern eines, das von sich aus bereits eine besondere Eignung für die Bezeichnung konkreter Gegenstände mit sich bringt, das somit per se einen semantischen Gehalt besitzt. Die generative Grammatikschreibung hat mit diesem Verständnis weitgehend gebrochen und eine nicht-symbolische Syntax und Sprachtheorie entwickelt, in der Bezüge zwischen Form und Inhalt keine zentrale Rolle mehr spielen und Konstruktionen allenfalls als Epiphänomene allgemeiner Regeln anzusehen sind. In Opposition zum generativen Paradigma haben Sprachwissenschaftler wie Langacker, Fillmore, Lakoff und Goldberg und teilweise auch Jackendoff in den 1980er und 1990er Jahren wiederum den Anschluss an das traditionelle Verständnis von Grammatik als einem Inventar von Zeichen, d. h. von Form-InhaltPaarungen, gesucht (zu diesem „Paradigmenwechsel rückwärts“ s. auch Webelhuth 2011: 175f.).

Um die Unterschiede in der Herangehensweise an zentrale sprachliche Erscheinungen deutlich zu machen, die zwischen der generativen Beschreibungstradition und konstruktionellen Ansätzen bestehen, sei hier auf Goldbergs Auseinandersetzung mit Argumentstrukturen von Verben verweisen. In der generativen Tradition werden die Argumente, die in einem Satz realisiert sind, als Projektionen der $\theta$-Rollen des Verbs aus dem Lexikon beschrieben (vgl. Chomsky 1981). Vgl. dazu etwa die beiden Sätze in (20).

(20) a. Hans backt einen Kuchen.

b. Hans backt seiner Frau einen Kuchen.

Die Argumentstruktur in (20a) ist im Rahmen dieses Beschreibungsansatzes problemlos als Projektion der $\theta$-Rollen von backen zu erklären. Ein Satz wie (20b) hingegen bereitet Schwierigkeiten, da backen eine zusätzliche Bedeutungskomponente FÜR JEMANDEN aufweist, die im Lexikoneintrag von backen zunächst nicht vorgesehen zu sein scheint. Um das Projektionsprinzip zu wahren, wird eine lexikalische Regel postuliert, die backen (b) aus backen (a) ableitet (vgl. Pinker 1989; Levin/Rappaport Hovav 1995).

In der Konstruktionsgrammatik dagegen wird die zusätzliche Bedeutungskomponente in (b) nicht als Output einer lexikalischen Regel erklärt. Stattdessen wird 
angenommen, dass es eine Ditransitivkonstruktion [Subj V Obj1 Obj2] gibt, welche die betreffende Bedeutung enthält. Diese Konstruktion stellt ein syntaktisches Muster dar, das mit einer relativ allgemeinen Bedeutung gekoppelt ist. Im Fall der Ditransitivkonstruktion handelt es sich um die schematische Bedeutung TRANSFER: „the agent [...] acts to cause transfer of an object to a recipient“ (Goldberg 1995: 32), vgl. auch die Notation der Ditransitivkonstruktion in (21), hier in Anlehnung an die Notierungen bei Croft (2003: 56).

\section{[[Subj V Obj1 Obj2]/[CAUSE RECEIVE <AGT REC PAT>]]}

Die These, dass die Ditransitivkonstruktion tatsächlich Transferbedeutung trägt, stützt sich auf Beipiele wie (20b), in denen ein Verb wie backen vorliegt, das in der Regel keine Transferlesart aufweist. Die Transferlesart kommt aus konstruktionsgrammatischer Sicht erst dann zustande, wenn das Lexem backen in die Ditransitivkonstruktion integriert wird. Die Bedeutungskomponente TRANSFER geht somit nicht auf die Semantik des Verbs, sondern auf die Semantik der Konstruktion zurück. Aufgrund der Annahme, dass die TRANSFER-Bedeutung durch die Konstruktion und nicht durch das Verb der Satzbedeutung beigesteuert wird, kann auf lexikalische Regeln, die das Verb backen in (20b) aus backen in (20a) generieren, verzichtet werden. Die ad-hoc-Postulierung idiosynkratischer und damit wenig plausibler Verbbedeutungen, die die Beschreibung mittels lexikalischer Regeln mit sich bringt - für backen in (20b) etwa „X INTENDS to CAUSE Y to HAVE Z BY BAKING“ -, entfällt damit ebenfalls (vgl. Goldberg 1995: 9). Darin kann ein Vorteil des konstruktionsgrammatischen Ansatzes gegenüber dem regelbasierten Ansatz gesehen werden (vgl. weiter Goldberg 1995: 9-23).

Die Konstruktionsgrammatik erhebt allerdings nicht nur den Anspruch, einzelne Erscheinungen wie das Auftreten des indirekten Objekts in (20b) besser als die herkömmlichen generativen Beschreibungen zu erklären. Sie versucht vielmehr eine umfassende Alternative zur generativen Sprachtheorie zu entwickeln. Die zentralen Positionen der Konstruktionsgrammatik können daher besonders prägnant in ihrer Abgrenzung gegenüber dem generativen Paradigma charakterisiert werden. Folgende Grundannahmen sind für die meisten der heute geläufigen Versionen der Konstruktionsgrammatik prägend (als wichtigste Vertreter wären zu nennen Lakoff 1987; Langacker 1987; Kay 1997; Kay/Fillmore 1999; Goldberg 1995; 2006; Croft 2001): ${ }^{79}$

79 Übersichtsdarstellungen zur Konstruktionsgrammatik bieten Goldberg (2003), (2013), Croft/Cruse (2004: 225-290), Fischer/Stefanowitsch (2006), Engelberg/Holler/Troost (2011) sowie Lasch/Ziem (2013, besonders 31-76). Die folgende Skizze stützt sich in vielen Punkten auf 
- Der Konstruktionsgrammatik zufolge handelt es sich bei allen sprachlichen Einheiten vom Morphem über das Wort bis hin zu Satzmustern um konventionalisierte Kombinationen von Form und Bedeutung, d. h. um Zeichen im Sinne de Saussures. Die Konstruktionsgrammatik ist somit, wie bereits erwähnt, zeichenbasiert bzw. symbolisch. Unter „Form“ werden dabei alle phonologischen, morphologischen, syntaktischen und prosodischen Strukturen verstanden, „Bedeutung“ umfasst sowohl lexikalische Semantik als auch Pragmatik.

- Gegenstand der grammatischen Beschreibung ist nicht ein privilegierter, als „Kern“ angesehener Teilbereich der sprachlichen Erscheinungen. Die Konstruktionsgrammatik versucht vielmehr die Gesamtheit der sprachlichen Ausdrucksformen zu erfassen und bezieht dabei auch den weiten Bereich der Idiome und Kollokationen in ihre Überlegungen ein. Die Konstruktionsgrammatik verfährt somit holistisch.

- Die Konstruktionsgrammatik folgt dem Motto „What you see is what you get“. Außer der oben beschriebenen Ebene der Form und der Bedeutung werden daher keine weiteren Ebenen (etwa im Sinne einer D-Struktur) angesetzt. Phonologisch leere Elemente werden nach Möglichkeit nicht postuliert. Die Konstruktionsgrammatik kann somit als monostratal bezeichnet werden.

- Die Konstruktionen einer Einzelsprache werden als taxonomisches Netzwerk modelliert. Die Konstruktionen, die dieses Netzwerk bilden, sind durch Vererbung formaler und inhaltlicher Eigenschaften miteinander verbunden. Für den Bestand an Konstruktionen, den eine Sprache aufweist, wird somit keine spezielle Organisationsform postuliert, sondern davon ausgegangen, dass die Form-Bedeutungs-Paarungen prinzipiell nicht anders als das Weltwissen organisiert sind. In diesem Sinne ist die Konstruktionsgrammtik kognitiv orientiert.

- Die Konstruktionsgrammatik ist gebrauchsbasiert (usage-based), d. h. die Repräsentation sprachlicher Information ist nicht unabhängig von ihrem Gebrauch in der Kommunikation zu sehen, wie strukturalistische und generative Modelle annehmen. Sprachliches Wissen umfasst somit gleichermaßen konkrete Gebrauchsinstanzen wie abstrakte Repräsentationen. ${ }^{80}$

die genannten Arbeiten, hier vor allem Goldberg (2013: 15f.); daher wird auf Einzelnachweise in der Regel verzichtet.

80 Zu diesem Punkt speziell Goldberg (2013: 15). Die Gebrauchsbasierung der Konstruktionsgrammatik wird gelegentlich auch dahingehend interpretiert, dass diese nur korpuslinguistisch 
Obgleich die Konstruktionsgrammatik sich mit diesen Postulaten deutlich vom generativen Mainstream absetzt, teilen beide Herangehensweisen grundlegende Erkenntnisziele: Beide Modelle stimmen darin überein, dass Sprache als Teil des kognitiven Systems beschrieben werden muss - sei es nun als autonomes oder als integriertes Teilsystem. Dazu gehören besonders auch eine Erklärung der sprachlichen Kreativität, d. h. der Fähigkeit, neue Äußerungen hervorzubringen, sowie eine nicht-triviale Theorie des Spracherwerbs (vgl. Goldberg 2003a: 219). ${ }^{81}$

Trotz weitgehender Übereinstimmungen zwischen ihren einzelnen Ausprägungen und Schulen ist die Konstruktionsgrammatik (noch) nicht als homogenes Theoriegebilde anzusehen. Gegenwärtig zeichnen sich vielmehr im Wesentlichen zwei große Strömungen ab: die sog. „Berkeley School“ um Fillmore und Kay sowie die wesentlich in der Tradition der kognitiven Linguistik fußenden Ansätze von Langacker, Lakoff, Goldberg und Croft. Die kognitiv orientierten Konstruktionsgrammatiker sind insgesamt stärker an einer psychologisch realistischen Beschreibung interessiert als die von Kay/Fillmore entwickelte Konstruktionsgrammatik, die keine Aussage über Repräsentationen im Geist des Sprechers macht (vgl. explizit Kay 1997: 129). Die Berkeley-School legt auch größeren Wert auf eine Formalisierung ihrer Beschreibungen und nutzt dabei Attribut-Wert-Matrizen, wie sie auch in der HPSG (Pollard/Sag 1994) üblich sind. Die kognitiv orientierten Strömungen verhalten sich dagegen im allgemeinen zurückhaltender gegenüber einer durchgehenden Formalisierung ihrer Analysen, da Formalismen aufgrund der Zwänge und Beschränkungen, die sie der linguistischen Untersuchung auferlegen, häufig eher als Hindernis denn als Hilfe bei der adäquaten Beschreibung linguistischer Daten empfunden werden (vgl. Croft 2001: xiii, 3f.; Stefanowitsch 2006: 153). Einige Grundüberzeugungen der Konstruktionsgrammatik finden sich übrigens auch in den Arbeiten von Jackendoff (1997; 2002; 2008). Der konstruktionelle Ansatz ist bei Jackendoff freilich in eine umfassende Sprachtheorie eingebettet, die letztlich doch weitgehende Übereinstimmungen mit dem generativen Paradigma erkennen lässt (vgl. auch Kapitel 8).

zu betreiben sei; kritisch dazu sowie allgemein zum Verhältnis von Konstruktionsgrammatik und Korpuslinguistik Welke (2019: 52).

81 Zur Frage, ob und, wenn ja, in welcher Hinsicht die Konstruktionsgrammatik als Teil der „generative enterprise“ verstanden werden kann, vgl. die in Fischer/Stefanowitsch (2006: 12f.) referierten Positionen. 


\subsubsection{FVG im Kontinuum von Konstruktionen}

Als Konstruktionen sind - zumindest der verbreiteten Auffassung Goldbergs (1995) zufolge - grundsätzlich alle Kombinationen von Form und Bedeutung aufzufassen, deren Bedeutung bzw. Form nicht strikt auf der Basis anderer Konstruktionen vorhersagbar ist:

Any linguistic pattern is recognized as a construction as long as some aspect of its form or
function is not strictly predictable from its component parts or from other constructions
recognized to exist. (Goldberg 2003a: 219, vgl. Goldberg 1995: 13) ${ }^{82}$

So ist etwa die ditransitive Struktur [Subj V Obj1 Obj2] als Konstruktion zu beschreiben, da ihre Bedeutung, die als TRANSFER charakterisiert werden kann (s. o.), weder auf der Basis ihrer Teile noch einer anderen Konstruktion strikt vorhersagbar ist.

Als Konstruktionen kommen, wie bereits angedeutet, prinzipiell alle bedeutungstragenden Einheiten der Sprache vom Morphem bis hin zum syntaktischen Strukturmuster in Betracht. Auch die Phraseologismen gehören dazu, da diese hinsichtlich ihrer formalen und inhaltlichen Eigenschaften nur bedingt bzw. gar nicht vorhersagbar sind (vgl. auch Croft/Cruse 2004: 236-247).

Dass es sich bei FVG grundsätzlich um Konstruktionen im Sinne der Definition Goldbergs handelt, ist ohne Weiteres einzusehen: Die semantischen Interpretationsregeln, die z. B. für das Syntagma Antwort geben und seine Konstituenten gelten, stimmen mit den allgemeinen Interpretationsregeln von Antwort und geben gleich in mehrfacher Hinsicht nicht überein und sind daher nicht vorhersagbar: Zunächst kodiert geben im Regelfall nur den Transfer konkreter Objekte, und Antwort ist normalerweise nicht Objekt eines konkreten Transfervorgangs. Das Syntagma Antwort geben erfordert somit eigene semantische Interpretationsregeln. Sodann geht das Verb geben nicht in der Weise in die Gesamtbedeutung des FVG ein, wie es beispielsweise in der Kombination Buch geben der Fall ist. Geben ist vielmehr, wie mehrfach angedeutet, semantisch leer.

82 In einer erweiterten Definition von ,Konstruktion‘ zählt Goldberg (2006: 5) neben den Verbindungen, die nicht kompositional sind, auch vollständig vorhersagbare Muster zu den Konstruktionen, sofern diese häufig genug sind, um als Bestandteile der Speicherkomponente angesehen zu werden. 
(22) ein Buch geben EIN BUCH GEBEN

(23) Antwort geben $\frac{\text { ANTWORTEN }}{\square}$

Somit ist es letztlich die in Kapitel 3 dargelegte Tatsache, dass die Nominalbedeutung die Gesamtbedeutung impliziert, selbst, die diese Fügungen als Konstruktionen erweist, ist doch ein solches Implikationsverhältnis bei anderen NomenVerb-Kombinationen nicht gegeben.

Tab. 3: FVG im Kontinuum der Konstruktionen

\begin{tabular}{|c|c|c|}
\hline Konstruktionstyp & Untertyp & Beispiel \\
\hline \multirow[t]{3}{*}{$\begin{array}{l}\text { (1) Idiom mit vollständiger } \\
\text { lexikalischer Spezifikation }\end{array}$} & (a) Sprichwort & $\begin{array}{l}\text { Morgenstund hat Gold im Mund. } \\
\text { Was muss, das muss. }\end{array}$ \\
\hline & $\begin{array}{l}\text { (b) feste Phrase } \\
\text { (Burger 2010: 39) }\end{array}$ & $\begin{array}{l}\text { Deiktische, anaphorische Phrase, } \\
\text { z. B. Das/diese Unverschämtheit } \\
\text { schlägt dem Fass den Boden aus! }\end{array}$ \\
\hline & $\begin{array}{l}\text { (c) idiomatische NP } \\
\text { (mit syntaktischer An- } \\
\text { schlussmöglichkeit) }\end{array}$ & schwarzes Gold \\
\hline $\begin{array}{l}\text { (2) Idiom mit vollständiger } \\
\text { lexikalischer Spezifikation } \\
\text { und offener Valenz }\end{array}$ & idiomatische VP ${ }^{83}$ & $\begin{array}{l}\text { (Jmd.) zieht (jmdm.) die Würmer aus } \\
\text { der Nase }\end{array}$ \\
\hline \multirow[t]{2}{*}{$\begin{array}{l}\text { (3) Idiom mit partieller le- } \\
\text { xikalischer Spezifikation }\end{array}$} & $\begin{array}{l}\text { (a) phraseologischer Teil- } \\
\text { satz (Richter/Sailer 2009) }\end{array}$ & $\mathrm{V}$, bis der Arzt kommt \\
\hline & $\begin{array}{l}\text { (b) „Phraseoschablone“: } \\
\text { mit Synsemantika }\end{array}$ & $\mathrm{N}$ für $\mathrm{N}$; Der und $\mathrm{N}$; $\mathrm{N} \mathrm{V}_{\mathrm{i}}$ und $\mathrm{V}_{\mathrm{i}}$ \\
\hline $\begin{array}{l}\text { (4) formale Konstruktion } \\
\text { (ohne lexikalische Spezifi- } \\
\text { kation) (vgl. Jackendoff } \\
\text { 1997: 172) }\end{array}$ & $\begin{array}{l}\text { (a) Phrasenschema } \\
\text { (b) Satzschema }\end{array}$ & $\begin{array}{l}\text { NNGen }_{\text {(der König der Könige) }} \\
\text { [NP V NP PP/AP] } \\
\text { kausatives Resultativum } \\
\text { (jmdn. müde reden) }\end{array}$ \\
\hline
\end{tabular}

Da die Konstruktionsgrammatik ein breites Übergangsfeld zwischen (mehr oder weniger) vorhersagbaren und nicht vorhersagbaren Bildungen annimmt, müsste

83 Fillmore/Kay/O’Connor (1988: 505) unterscheiden grundsätzlich zwei Idiomtypen: lexikalisch gefüllte Idiome („substantive or lexically filled idioms“) und lexikalisch offene Idiome („formal or lexically open idioms“). Zu den lexikalisch offenen Idiomen zählen sie auch solche, die einzelne Synsemantika (vor allem Konjunktionen) enthalten. 
es vor diesem theoretischen Hintergrund möglich sein, auch die FVG in diesem Feld genauer zu verorten. Grundlage dafür kann die Skala in Tab. 3 sein, die den einschlägigen Übergangsbereich zwischen Phraseologie und Syntax modelliert. Die konstruktionelle Skala reicht hier von phraseologischen Verbindungen, deren Wortbestand vollständig festgelegt ist (Typ 1), über solche, die einzelne, mehr oder weniger frei besetzbare Leerstellen offenlassen (Typ 2 und 3), bis hin zu Kombinationsmustern, die lediglich syntaktische Spezifikationen, jedoch keine phonologische Füllung vorgeben (Typ 4).

Wenn man FVG in dieses Kontinuum zwischen phonologisch vollständig spezifizierten und phonologisch nicht spezifizierten Verbindungen einzupassen versucht, so kommt am ehesten eine Position zwischen 3a und 3b in Betracht: FVG enthalten ein festgelegtes Verb sowie eine Leerstelle für eine NP bzw. PP; damit verfügen sie über weniger lexikalisches Material als ein phraseologischer Teilsatz wie V, bis der Arzt kommt. Im Unterschied zu den Phraseoschablonen, die nur synsemantische Wörter enthalten (Präpositionen, Konjunktionen und Artikelwörter), verfügen sie immerhin über ein Verb. FVG sind damit nicht mit Phraseoschablonen gleichzusetzen (s. auch Abschnitt 4.2.1), sie gehören in dieser Skala aber in der Tat in die Nähe der Phraseoschablonen.

Mit dieser Einordnung ist zwar eine gewisse Klarheit im Hinblick auf die Position der FVG zwischen Phraseologie und Syntax gewonnen, eine entscheidende Frage ist aber noch nicht beantwortet: Wie frei besetzbar ist die Leerstelle in FVG? Handelt es sich überhaupt um eine Leerstelle? Sind nicht FVG möglicherweise als einzelne Phraseologismen repräsentiert, zwischen denen allenfalls Ähnlichkeitsrelationen bestehen? Diese Fragen sind im anschließenden Kapitel zu klären. 